\title{
Las resistencias del profesorado universitario a la utilización de las tecnologías digitales
}

\author{
Cristina Mercader Juan
}

Universidad Autónoma de Barcelona

\section{RESUMEN}

El estudio desarrollado centra su foco de atención en el profesorado universitario y en los motivos por los que no utilizan las tecnologías digitales de manera habitual como herramienta de enseñanza-aprendizaje en el aula universitaria. El objetivo principal es identificar cuáles son las resistencias del profesoradoa la utilización de tecnologías digitales en la Facultad de Ciencias de la Educación de la Universidad Autónoma de Barcelona. Se utiliza una metodología mixta secuencial. En primer lugar, se realiza un cuestionarioal profesorado de la Facultad (N=78) y, a posteriori, entrevistas a profesores y gestores de la universidad. Los resultados muestran que el hábito, la gestión de la innovación, la falta de formación, el trabajo a contrarreloj y la inversión en actualización son las principales resistencias. En conclusión, se determina que la utilización de las tecnologías digitales es escasa, que su empleo no depende tanto de la edad o estabilidad laboral, sino de la predisposición y autopercepción de las propias competencias digitales; y que una de las mayores barreras es la falta de planificación y evaluación sobre cómo las tecnologías digitales se deben institucionalizar en la universidad.

Palabras Clave: TIC, tecnologías digitales, tecnología educativa, educación superior, resistencia al cambio.

\section{Resistances to use digital technologies in University teachers}

\section{ABSTRACT}

The study focuses on university teaching staff and the reasons why they do not regularly use digital technologies as a teaching-learning tool in the university classroom. The main objective is to identify the resistances to the use of digital technologies of the instructors of the Faculty of Educational Sciences of the Autonomous University of Barcelona. A sequential mixed methodology is used. First, we apply a questionnaire to instructors $(\mathrm{N}=78)$ and, afterwards, we carry on interviews with teachers and managers. The results show habit, management of innovation, lack of training, clockwork and investment in updating as the main resistances to the use of digital technologies. In conclusion, it is determined that the use of digital technologies is scarce, that their use does not depend so much on age or job stability, but on the predisposition and self-perception of knowledge of digital technologies, as well as the lack of planning and evaluation in the institutionalization of change are two of the biggest barriers.

Keywords: ICT, digital technologies, educational technology, higher education, resistance to change.

\section{Introducción}

La investigación propuesta se centra en el profesorado universitario y en los motivos por los cuales éstos no utilizan más las tecnologías digitales como herramientas de docencia. Actualmente, las tecnologías evolucionan a gran velocidad en la sociedad teniendo un impacto en la cultura universitaria (Falco, 2017) y por ello es importante que el profesorado esté en constante reciclaje para que devengan un facilitador del proceso de enseñanza-aprendizaje (Valdés, Ramírez, Moreno, Núñez y Lugo, 2012) ya que aportan inmediatez en su seguimiento, personalización y retroalimentación de las actividades académicas (Moreno y Rochera, 2015).
El contexto del estudio es la educación superior, concretamente, el profesorado universitario que imparte clases a los futuros maestros de educación primaria. El motivo de esta selección ha sido por el papel relevante que éstos tienen en la educación al ser responsables de la formación de los maestros. Johnson et al., (2016, p.4), en el Horizon Report sobre Educación Superior, apuntan que la construcción del conocimiento debe ser apoyada por las TIC porque éstas ayudan a diseñar, crear y trabajar de manera colaborativa. Además, dado que se debe tener en cuenta la idiosincrasia de las instituciones en cuanto se analiza cuál es la integración que estas hacen de las tecnologías digitales (Salinas, 2004), el presente estudio se enmarca en el caso de la Universitat Autònoma de Barcelona, concretamente en la Facultad de Ciencias de la Educación. 


\section{Problema de investigación}

El problema de investigación nace de la necesidad de mejorar la utilidad que el profesorado da a las tecnologías digitales actualmente. Publicaciones como la de Sigalés (2004) o Cejudo (2008) destacan la necesidad de la formación del profesorado como elemento clave para mejorar la utilización de las tecnologías digitales en el aula. En la misma línea, Prendes y Castañeda (2010) realizan un estudio en el que se pone en relieve el bajo nivel del profesorado universitario en TIC. Asimismo, San Martín, Jiménez y Jerónimo (2016) en su investigación sobre las guías docentes afirman que se emplean poco las tecnologías. Sin embargo, los estudiantes universitarios parecen utilizarlas con objetivos académicos y teniendo una alta valoración sobre la importancia de las mismas para su desarrollo profesional (Bonilla-del-Río, Diego-Mantecón y Lena-Acebo, 2018).

Pero, si es un problema de formación, ¿por qué sigue sin resolverse? ¿Y si no se trata sólo de formación? ¿Puede el profesorado estar suficientemente formado y aun así no quiera utilizar más las tecnologías digitales en el aula?

A partir de estas reflexiones, surge la necesidad de llevar a cabo un estudio en profundidad sobre las resistencias a las tecnologías digitales para comprobar la naturaleza de dichas barreras.

Así pues, la pregunta que se plantea es ¿Por qué el profesorado universitario no utiliza más las tecnologías digitales en el proceso de enseñanza-aprendizaje?

Algunos estudios hacen mención de algunos motivos de la falta de utilización de las tecnologías digitales. La British Educational Communications and Technology Agency (BECTA, 2004) y Kalembera y Majawa (2015) señalan la importancia del reconocimiento de las barreras existentes en un contexto determinado, ya que éstas afectan al profesor y a la organización; y es el primer paso necesario para eliminarlas. Sin este análisis de los motivos, no se consigue atenuar o eliminar las barreras, ni conseguir una integración completa. En el caso del estudio de Duart y Lupiáñez $(2005$, p.24) afirman que "se aprecia una importante resistencia institucional, no necesariamente derivada de la resistencia de las personas que forman parte de la institución, sino de la rigidez estructural".

Por otro lado, en el estudio de Prendes (2010) se indica que hay un alto conocimiento en TIC pero sólo en ciertas herramientas: el correo electrónico, presentaciones visuales, motores de búsqueda y foros. En cambio, un porcentaje bastante bajo utiliza las redes sociales, microblogging, herramientas de intercambio de archivos, lectores de RSS, herramientas de publicación en red, lifestreaming, mundos virtuales o videoconferencia. El motivo es el propio desconocimiento: "el desconocimiento redunda en muchas ocasiones en un menor uso de las TIC en el aula" (Prendes, 2010, pp.151-152).

Estudios más recientes apuntan a un aumento del uso de redes por parte del profesorado (Vázquez y Cabero, 2015). Sobre Facebook (Cartagena, 2016; Gewerc, Montero y Lama, 2014) y Twitter (Maor y Currie, 2017) se señalan los beneficios académicos y relacionales de las redes sociales, pero siguen siendo prácticas mayoritariamente esporádicas o provenientes únicamente de algunos profesores (Gómez, Roses y Farias, 2012).

UNESCO (2011, p.71) analiza los obstáculos en la integración de las tecnologías digitales como los prejuicios personales, los diferentes puntos de vista y perspectivas sobre la docencia, el rechazo e inhabilidad de considerar nuevas ideas o la ausencia de programas de aprendizaje profesionales.

Por lo tanto, es evidente que la resistencia a la utilización de las tecnologías digitales sigue siendo un problema existente en las aulas universitarias y que aunque se han propuesto posibles soluciones, todavía no se ha conseguido superarlo.
En relación al proceso de cambio, es esencial tener en cuenta las fases de aplicación. Curry (1992) las organiza en: movilización, implantación e institucionalización. Por su parte, Gairín y Rodríguez-Gómez (2011) revisan los tres niveles de implicación:

(1) La reconstrucción del cambio educativo promovido e impulsado externamente.

(2) La creación de condiciones internas.

(3) La efectividad del cambio relacionado con su incidencia en las aulas, en las que se incluye modificaciones que afectan al clima y los procesos de enseñanza-aprendizaje.

Por otra parte, para realizar el cambio se debe conocer qué tipo de resistencias nos podemos encontrar para poder proponer los facilitadores más adecuados para paliarlas (Lewin, 1947) y entender estas resistencias como algo natural al proceso de cambio y tomar consciencia de las dinámicas de poder que se generan en las organizaciones (Pieterse, Caniëls y Homan, 2012).

El tipo de resistencias que se pueden generar es diverso y por ello es necesario conocer las tipologías existentes ya que cada una requerirá de un facilitador adecuado para paliarla. Tejada (1998) divide las resistencias en 5 categorías que van desde el objeto más concreto al objeto más general (o macro): Individuales, Grupales, Proceso Instructivo, Sistema Educativo y Sistema Social. Cada una de estas resistencias, se concreta en subcategorías de manera que, finalmente, se pueden llegar a detectar 31 tipos de resistencias al cambio. Sin embargo, hay que tener en cuenta que pese a que se puede concretizar la tipología de resistencia, todas ellas son influidas por las demás y a su vez influyentes de las demás, de manera que se hace necesario el conocimiento de todas ellas para comprender la complejidad del cambio en cuestión.

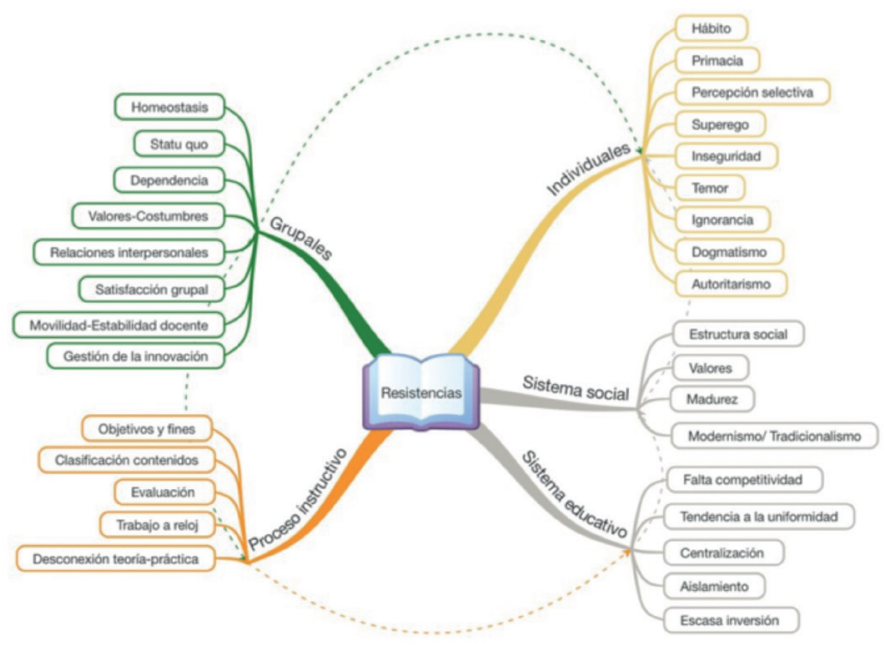

Figura 1. Clasificación de las resistencias al cambio (Fuente: Tejada, 1998).

La categorización propuesta por Robbins y Judge (2013) es más sintética. Para los autores, las resistencias se generan a partir de dos fuentes: la fuente individual y la fuente organizacional. La fuente individual es toda aquella que surge a partir del propio individuo sujeto a un proceso de cambio y sus resistencias concretas se derivarían del (1) hábito, (2) seguridad, (3) miedo a lo desconocido, (4) factores económicos, (5) procesamiento selectivo de la información. En cambio, la fuente organizacional incluye aquellas resistencias derivadas de las situaciones organizacionales dentro de una institución como pueden ser (1) la inercia estructural, (2) la inercia del grupo, (3) una idea limitada 
del cambio, (4) la amenaza de los expertos, y (5) la amenaza a las relaciones de poder establecido.

En todo caso, las resistencias al cambio son múltiples y delimitarlas es una tarea necesaria para poder identificarlas en cada contexto y poder proponer medidas para paliarlas.

\section{Metodología}

La presente investigación persigue conocer las opiniones y percepciones que el profesorado tiene sobre la utilización de las tecnologías digitales.A partir del análisis de la literatura, se ha identificado que se carece de estudios que identifiquen de manera práctica y concreta los motivos por los que no se están utilizando las tecnologías educativas de manera generalizada en la educación superior. En las diversas publicaciones analizadas, se señalan algunos factores o barreras que pueden estar impidiendo esta generalización, pero no se presentan estudios en contextos concretos. Así, seplanteandos objetivos:

- Identificar las resistencias del profesorado universitario a la utilización de las tecnologías digitales en la docencia.

- Corroborar si la resistencia a las tecnologías digitales tiene relación directa con factores personales y profesionales.

Para la consecución de los objetivos, se ha realizado un estudio de caso con una metodología mixta secuencial CUAN cual. Primeramente, a partir de la aplicación del cuestionario cuantitativo creado ex profeso se recogieron las percepciones del profesorado sobre el objeto de estudio con un muestreo no aleatorio disponible. El cuestionario está validado por constructo a partir del análisis del marco teórico y previos estudios similares, expuesto en el apartado anterior. Seguidamente, se realizó una validación de su contenido por parte de seis jueces teóricos (expertos/as en la materia) y seis jueces prácticos (profesores/as de universidad), los cuales valoraron su univocidad, su pertinencia y su importancia. A partir de estas aportaciones, se ajustaron los ítems convenientemente.

Finalmente, a posteriori de la recogida de los datos se ha comprobado la consistencia interna del cuestionario a partir de la medición de su Alfa de Cronbach, el cual obtiene una puntuación de 0.824 .

El cuestionario constaba de dos partes: Preguntas sobre el perfil del docente participante para poder describir la población a nivel estadístico (edad, formación, categoría profesional, etc.);y afirmaciones sobre las cuales el profesor debía señalar su grado de acuerdo sobre la utilización de las tecnologías digitales en la docencia universitaria,así como aspectos sobre las percepciones del cambio.

Tras el análisis de las respuestas recibidas, se desarrolló el guion de la entrevista que se realizó a diferentes profesores responsables de gestión universitaria.

\section{Población y muestra}

La investigación se focaliza en el caso del profesorado universitario que imparte docencia a los futuros maestros de educación primaria de la Universidad Autónoma de Barcelona en el 2014.

La participación en el cuestionario permitió obtener el 95\% de confianza de los datos muestrales (con un error máximo del $8 \%$ ). La muestra mantiene la relación de género existente en la Facultad (66\% mujeres) así como la estabilidad del profesorado (53\% en posición estable). La media de edad de los sujetos par- ticipantes es de 47,33 años $(S=9,96)$ y 14,82 años de media de experiencia como profesor universitario $(S=10,36)$.

En cuanto a la utilización que hacen de las Tecnologías de la Información y la Comunicación, se observan diferencias entre el uso personal $(83,3 \%)$ y el profesional $(62,3 \%)$. La mayor parte de la muestra afirma haber realizado cursos sobre tecnologías $(75,6 \%)$ pero menos de la mitad de ellos, afirma que hayan sido satisfactorios (48,7\%). La percepción que el profesorado tiene sobre su nivel de conocimiento de las tecnologías es claramente de nivel medio (56\%) frente al nivel inicial $(5,3 \%)$, nivel avanzado $(26,7 \%)$ y nivel competente $(12 \%)$.

\section{Resultados}

Los hallazgos que se presentan a continuación parten del análisis de los cuestionarios, las preguntas abiertas y las entrevistas. Dada la tipología de método aplicado (mixta secuencial CUAN cual), los resultados más predominantes son los obtenidos a partir del cuestionario. Las entrevistas y preguntas abiertas complementan los hallazgos cuantitativos y los corroboran, en mayor parte, permitiendo la triangulación de los datos.

Los resultados estadísticos se analizaron con el programa SPSS versión 15.0. El análisis descriptivo se ha realizado a partir de la observación de las medias y el inferencial se ha obtenido aplicando las pruebas ANOVA y Bonferroni en aquellas variables politómicas: edad, experiencia docente y nivel de conocimiento, con el objetivo de identificar las diferencias significativas entre grupos.

\subsection{Análisis de las resistencias a la utilización de las Tecnolo- gías Digitales}

Los hallazgos muestran que el hábito, la gestión de la innovación, la falta de formación, el trabajo a contrarreloj y la inversión en actualización son las principales resistencias.

Los resultados estadísticos del cuestionario señalan el hábito $(\mathrm{M}=3,66, \mathrm{SD}=1,04)$, el trabajo a contrarreloj $(\mathrm{M}=3,56, \mathrm{SD}=$ $0,95)$ y la gestión de la innovación $(\mathrm{M}=3,24 ; \mathrm{SD}=1,07)$ como las resistencias más recurrentes entre el profesorado, mientras que la regresión $(\mathrm{M}=1.55, \mathrm{SD}=0.75)$, el temor $(\mathrm{M}=1,46, \mathrm{SD}=0,72)$ y el superego $(\mathrm{M}=1,88, \mathrm{SD}=1,02)$ las resistencias prácticamente inexistentes entre el profesorado.

Los resultados de las preguntas abiertas del cuestionario permiten corroborar que estas resistencias identificadas también son señaladas por el profesorado. Sin embargo, se incluyen también la falta de formación $(\mathrm{N}=40)$ y la falta de recursos $(\mathrm{N}=$ 18), aunque también aparecen otras como la propia resistencia al cambio $(\mathrm{N}=10)$ y la movilidad y falta de estabilidad del profesorado $(\mathrm{N}=4)$.

En la misma línea están los resultados de las entrevistas tanto con profesores como con responsables de la Facultad. Las resistencias que señala el profesorado son la falta de formación, la costumbre o hábito, la falta de calidad de las herramientas, el interés del profesorado y su actitud, el exceso de trabajo y que el proceso de cambio es lento.

Seguramente, a la Facultad todavía le falta bastante para poder tener todas las instalaciones y todos los aparatos necesarios para utilizarlas, aunque es verdad que el profesorado tampoco le saca el máximo rendimiento a lo que ya tenemos. (Vicedecana)

Yo creo que hay profesores que no las utilizan por rutina, porque cambiar cuesta y porque creo que hasta que no lo utilizas, no te das cuenta de lo potente que es. (Jefe de Departamento2) 


\subsection{Análisis de las diferencias en función de los rasgos persona- lesy profesionales}

Para analizar las diferencias en la utilización de las tecnologías digitales, se ha comparado los resultados a partir del género, edad, experiencia y conocimiento de las Tecnologías Digitales.

En el caso del género y la edad, no se observan diferencias significativas entre los grupos (hombre-mujer; joven-mediana edad-mayores) o la experiencia docente (principiante, madurez y veterano), aunque en esta última sí se observan algunas diferencias a destacar.

En la Figura 2 se observa como a nivel de utilización de las Tecnologías Digitales en la docencia, el profesor veterano (más de 6 años de experiencia) es aquel que las utiliza más (66,7\%) seguido del que se encuentra en el periodo de madurez (entre 6 y 15 años de experiencia, un 61,6\% bastante o mucho). El profesorado novel o principiante (menos de 6 años de experiencia) es aquel que utiliza menos las tecnologías en la docencia, pues ninguno de ellos indica utilizarlas mucho, aunque el 55,6\% las utilice bastante.

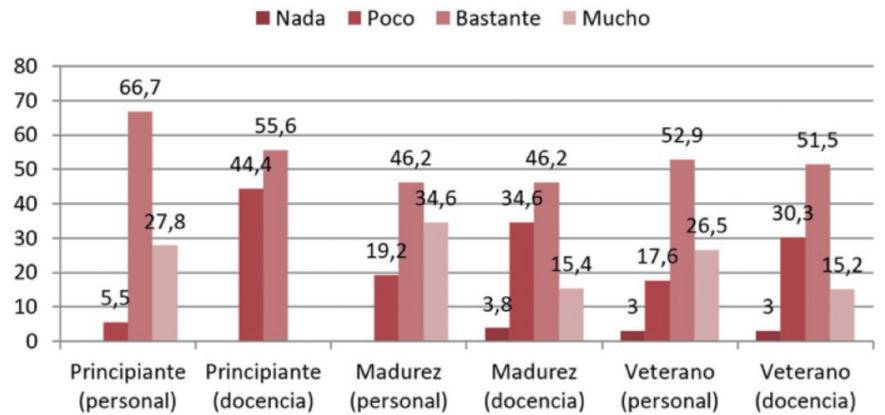

Figura 2. Utilización de las Tecnologías Digitales a nivel personal y docencia según la experiencia docente. Fuente: Elaboración propia

En el análisis cualitativo de los instrumentos, no aparecen el género o la experiencia. Sin embargo, tanto en las preguntas abiertas como en las entrevistas, el profesorado sí identifica que la edad es un factor que puede estar influyendo en la resistencia al uso de tecnologías digitales.

A ver yo creo que puede haber varios motivos, seguramente una cuestión generacional, es decir, si pudiésemos analizar esos resultados en relación a la edad de las personas seguramente veríamos tres grandes ámbitos que necesariamente no tiene que ser vinculado a la edad, pero yo creo que es un factor importante. (Secretario de Departamento1)

Estadísticamente, dónde se observan diferencias significativas es en la utilización de las tecnologías en la docencia según el conocimiento de las mismas ( $\mathrm{p}=0,000)$. Para identificar cuáles son los grupos que presentan mayores diferencias, se ha realizado la prueba posthoc de Bonferroni (Tabla 1).

Como se puede observar en la Tabla 1, existen diferencias significativas entre la utilización que hacen de las TIC los profesores con un nivel inicial frente a los que se encuentran en un nivel avanzado $(\mathrm{F}=14,714, \mathrm{p}=0,001)$ y frente a los que se encuentran en un nivel competente $(\mathrm{p}=0,000)$. Además, Bonferroni muestra que también hay diferencias significativas entre el grupo de conocimiento medio en TIC con el grupo de conocimiento avanzado $(p=0,011)$ y competente $(p=0,000)$. Estos resultados muestran que existe una relación entre el nivel de conocimiento de las tecnologías con el uso que se da en la docencia. Aquellos profesores que son más competentes en las tecnologías también son los que más las utilizan a nivel profesional.

En relación con los factores que inciden en la resistencia del profesorado a la integración de tecnologías, los resultados de la prueba ANOVA, muestran que no hay diferencias significativas de la tipología de resistencias según la edad y la experienciadocente. En el caso del género, la prueba estadística muestra diferencias significativas en el ítem 'Las tecnologías digitales representan una amenaza para la estabilidad del Departamento' asociada con la resistencia "amenaza y temor", los hombres afirman ser más resistentes a la utilización de las tecnologías digitales por amenaza y temor que las mujeres $(\mathrm{p}=0,025)$. También se observan

Tabla 1.

Comparación entre grado de conocimiento y utilización de las tecnologías digitales en la docencia

Variable dependiente: Utilización docencia

Bonferroni

\begin{tabular}{llccc}
\hline & Diferencia de & & & \\
(I) Grado conocimiento & (J) Grado conocimiento & medias (I-J) & Error típico & Sig. \\
\hline Inicial & Medio &,- 76220 &, 28838 &, 061 \\
& Avanzado & $-1,25000^{*}$ &, 30154 &, 001 \\
& Competente & $-1,80556^{*}$ &, 33083 &, 000 \\
Medio & Inicial &, 76220 &, 28838 &, 061 \\
& Avanzado &,$- 48780^{*}$ &, 15016 &, 011 \\
Avanzado & Competente & $-1,04336^{*}$ &, 20265 &, 000 \\
& Inicial & $1,25000^{*}$ &, 30154 &, 001 \\
& Medio &, $48780^{*}$ &, 15016 &, 011 \\
Competente & Competente &,- 55556 &, 22098 &, 085 \\
& Inicial & $1,80556^{*}$ &, 33083 &, 000 \\
& Medio & $1,04336^{*}$ &, 20265 &, 000 \\
\hline
\end{tabular}

* La diferencia de medias es significativa al nivel .05. 
diferencias significativas en relación al grado de conocimiento de las tecnologías digitales.

En el ítem 'En raras ocasiones hago mi trabajo con apoyo de las tecnologías digitales' $(\mathrm{F}=5,873, \mathrm{p}=0,001)$, asociado a la resistencia "Costumbres y hábitos" presenta diferencias significativas entre los profesores de nivel inicial con los de nivel avanzado $(p=0,007)$ y nivel competente $(p=0,002)$. Del mismo modo, se observan diferencias significativas con el mismo factor en el ítem 'Suelo evitar hacer cambios en relación a la utilización a las tecnologías digitales aunque sé que puede ser bueno para mí $(\mathrm{F}=5,843, \mathrm{p}=0,001)$, asociado a la resistencia "Inseguridad". Los profesores de nivel inicial en tecnologías digitales presentan resultados más resistencia que los profesores de nivel avanzado $(0,007)$ y nivel competente $(0,001)$.

Sin embargo, el factor que obtiene más diferencias significativas entre los grupos es la utilización en la docencia, en la que se identifican diferencias significativas entre grupos hasta en 5 tipologías de resistencias (Tabla 2):

Tabla 2.

ANOVA del factor utilización de las tecnologías digitales en la docencia en la tipología de resistencias a su utilización

\begin{tabular}{|c|c|c|c|c|c|c|}
\hline & & Suma de cuadrados & $\mathrm{gl}$ & Media cuadrática & $\mathrm{F}$ & Sig \\
\hline \multirow{3}{*}{$\begin{array}{l}\text { Si se ha tenido éxito sin utilizar las TIC es innecesa- } \\
\text { rio incorporarlas (Superego) }\end{array}$} & Inter-grupos & 11,026 & 3 & 3,675 & 3,901 & ,012 \\
\hline & Intra-grupos & 66,894 & 71 & ,942 & & \\
\hline & Total & 77,920 & 74 & & & \\
\hline \multirow{3}{*}{$\begin{array}{l}\text { Desconozco los objetivos que se persiguen con la } \\
\text { necesidad de una utilización generalizada de las } \\
\text { TIC en mi departamento (Objetivos) }\end{array}$} & Inter-grupos & 10,948 & 3 & 3,649 & 3,792 & 014 \\
\hline & Intra-grupos & 68,332 & 71 & ,962 & & \\
\hline & Total & 79,280 & 74 & & & \\
\hline \multirow{3}{*}{$\begin{array}{l}\text { Considero que podría incorporar metodologías } \\
\text { TIC a mis clases si tuviera más tiempo de planifica- } \\
\text { ción. (Trabajo a reloj) }\end{array}$} & Inter-grupos & 10,201 & 3 & 3,400 & 4,222 & ,008 \\
\hline & Intra-grupos & 58,786 & 73 & 805 & & \\
\hline & Total & 68,987 & 76 & & & \\
\hline \multirow{4}{*}{$\begin{array}{l}\text { Creo innecesario modificar mis estrategias didácti- } \\
\text { cas adaptándolas a las TIC (Homeostasis) }\end{array}$} & Inter-grupos & 8,570 & 3 & 2,857 & 3,241 & ,027 \\
\hline & Intra-grupos & & & & & \\
\hline & Total & 62,577 & 71 & 881 & & \\
\hline & & 71,147 & 74 & & & \\
\hline \multirow{3}{*}{$\begin{array}{l}\text { La utilización de las TIC no debería ser un criterio } \\
\text { de evaluación de calidad docente (Evaluación) }\end{array}$} & Inter-grupos & 12,172 & 3 & 4,057 & 3,847 & ,013 \\
\hline & Intra-grupos & 76,997 & 73 & 1,055 & & \\
\hline & Total & 89,169 & 76 & & & \\
\hline
\end{tabular}

Como se observa en la Tabla 2, las tipologías de resistencias "Superego", "Objetivos y fines", “Trabajo a reloj", "Homeostasis" y “Evaluación" presentan diferencias significativas entre el profesorado en función de la utilización de las tecnologías digitales en la docencia. Para identificar los grupos con diferencias significativas, se ha aplicado la prueba de Bonferroni. En el caso del Superego, es más resistente el que no las utiliza nunca frente a los que las utilizan poco $(p=0,033)$, bastante $(p=0,017)$ y mucho $(p=0,007)$. En cuanto a la resistencia por Objetivos y Fines, las diferencias significativas son entre los que las utilizan poco frente a los que las utilizan mucho $(p=0,027)$. En cuanto a la Homeostasis, es más común entre los que no utilizan nunca las tecnologías digitales en la docencia frente a los que la utilizan poco $(p=0,030)$, bastante $(\mathrm{p}=0,050)$ y mucho $(\mathrm{p}=0,020)$. Por último, es más habitual que la resistencia a la Evaluación del uso de las tecnologías digitales se dé en aquellos profesores que no las utilizan nunca en comparación a los que las utilizan bastante $(\mathrm{p}=0,022)$ y mucho $(\mathrm{p}=0,013)$.

\section{Conclusiones y Discusión}

Las conclusiones que se obtienen de esta investigación están directamente relacionadas con los objetivos del estudio. Para hacer el análisis, se parte de los objetivos de la investigación: identificación de resistencias y de factores condicionantes a esas resistencias.

\section{En relación con la tipología de resistencias}

Tras el estudio realizado, se entiende que el profesorado universitario que ha participado presenta resistencias a la utilización de las tecnologías digitales en la docencia, así lo corroboran las entrevistas y las preguntas abiertas. Pero el objetivo concreto en cuanto a la falta de utilización generalizada de las tecnologías digitales era conocer cuáles son las resistencias más fuertes en la Facultad. Así pues, principalmente, los motivos de la falta de utilización de las tecnologías digitales son el hábito de utilizar otro tipo de metodologías; la falta de formación en relación a la utilización en la docencia de las herramientas tecnologías digitales; el trabajo a reloj, la exigencia de otras tareas que no dejan tiempo a la actualización; la gestión de la innovación, el cómo desde la Facultad se ha gestionado la utilización de las tecnologías digitales y la inversión en actualización, la falta de recursos o actualización de infraestructuras.

Así pues, no se puede concluir que las resistencias a la utilización de las tecnologías digitales sean de una tipología concreta 
(individual, grupal, sistema instructivo, sistema educativo o sistema social) sino que las resistencias más marcadas se encuentran entre las diferentes tipologías.

Coincidimos con Salinas (2004, p.5) en cuanto a que "cada universidad debe responder desde su propia especificidad, partiendo del contexto en el que se halla" ya que cada contexto presenta rasgos y comportamientos distintos. Tal y como era de esperar, no se ha hallado ningún autor que considere las 5 principales resistencias a las tecnologías digitales detectadas en este estudio como las principales a nivel teórico. Sin embargo, en consonancia con los autores mencionados en el marco teórico, las resistencias señaladas en el estudio son tenidas en cuenta por uno $\mathrm{u}$ otro autor.

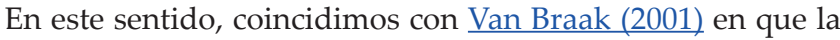
falta de infraestructuras, el software demasiado variado y la falta de tiempo son elementos que generan resistencias. Con Butler y Sellbom (2002) en que si el equipamiento es diferente, el software está desfasado, no hay soporte institucional y no se gestiona bien el tiempo, es más probable que existan resistencias a las tecnologías digitales. Con Rodríguez-Izquierdo (2010) en que los elementos a focalizar son la inversión en infraestructuras, la gestión del tiempo, la rutina, y la formación. Y con Tejedor, García-Valcárcel y Prada (2009) en que las resistencias se derivan de la falta de infraestructuras, el trabajo a reloj y la falta de conocimientos didácticos con tecnologías digitales por parte del profesorado.

\section{En relación a los factores personales}

Una de las suposiciones de las que se partía es que podían existir unos rasgos que influyeran en la utilización de las tecnologías digitales, pero a su vez, estos rasgos podían también influir en la tipología de resistencias a las tecnologías digitales. Tras el análisis de entrevistas, preguntas abiertas y cuestionario se concluye que el tipo de resistencia o su intensidad no depende de rasgos como la edad, el género, la experiencia o la estabilidad.

De todas formas, con las pequeñas diferencias que se han encontrado por los rasgos personales, se puede tener una idea de las características del perfil de profesor más resistente: hombre de más de 55 años, con más de 15 años de experiencia, con un empleo estable y que no suele utilizar las tecnologías ni a nivel personal ni profesional.

Por otra parte, en cuanto a la relación con las resistencias al cambio, los sujetos analizados parecen presentar más resistencias al cambio que a la utilización de las tecnologías digitales. Los valores que se observan en las resistencias al cambio son superiores a los valores que se observan sobre las resistencias a las tecnologías digitales. Por otra parte, a excepción de una tipología, la correlación entre las resistencias al cambio y las resistencias a las tecnologías digitales son bajas, muy bajas o nulas. Por eso se concluye que aunque el hecho de ser resistentes al cambio es un factor a tener en cuenta a la hora de introducir cambios en las metodologías, no es suficiente la correlación entre resistencias para afirmar que la falta de utilización de las tecnologías digitales se debe al propio rechazo al cambio.

En el presente estudio se ha concluido que no hay una clara influencia de rasgos personales con las resistencias a las tecnologías digitales. Esta afirmación coincide en parte con la de Van Braak (2001) el cual señalaba que "la actitud positiva hacia la utilización de las tecnologías digitales en el aula es más significativa que otros factores personales como la edad, el género, las aptitudes y la experiencia" (p54). Puesto que el presente estudio no ha profundizado en las actitudes del profesorado hacia las tecnologías digitales, no se puede afirmar que se coincide completamente con la conclusión de Van Braak (2001) pues no hay datos suficientes.
Finalmente, es necesario tener en consideración que pese a que la edad no ha resultado ser un factor que influya en las resistencias en el cuestionario, en las entrevistas se ha dejado entrever que los docentes sí que consideran que hay diferencias en función de la edad. Por lo tanto, esta aparente contraposición de perspectivas podría ser analizada con más profundidad en futuros estudios.

\section{En relación con los factores profesionales}

El estudio realizado apunta que la utilización y conocimiento de las tecnologías digitales no depende ni del género, ni de la experiencia docente, ni de la estabilidad laboral, ni de los cursos que ha recibido, ni de la edad. En cambio sí que se encuentra una relación directa con la percepción del grado de conocimiento de las tecnologías digitales. El profesorado que considera que tiene un nivel avanzado o competente utiliza más las tecnologías digitales en el aula; en cambio los que se consideran en un nivel medio las utilizan menos y los que se consideran a nivel inicial muy poco. Así, parece que cuanto más conocimiento se tiene, se pone en práctica con más asiduidad. Por lo tanto, se entiende que la competencia en tecnologías digitales es un elemento influyente en la posterior utilización de las tecnologías digitales.

Por otra parte, se observa un elemento paradójico en cuanto a la edad y la utilización de las tecnologías digitales en la docencia. El estudio parece indicar que los jóvenes utilizan mucho las TIC a nivel personal pero no las utilizan mucho en la docencia. En cambio, los mayores (más de 55 años) aunque las utilizan menos que los jóvenes a nivel personal, a nivel docente hay una parte que considera que las utiliza mucho. Estos resultados se pueden deber, como se ha comentado en el análisis de resultados, al hecho que al utilizarlas mucho a nivel personal, no perciben que las utilizan en el mismo grado a nivel docente. En cambio, un profesor que a nivel personal las utiliza poco y en la docencia aumenta su utilización, su percepción puede ser que las utiliza mucho, aunque en realidad en comparación con un joven puede que las utilice en menor medida. Es decir, el cuestionario medía con grados subjetivos, que pueden llevar a este tipo de contradicciones y requieren de un análisis del contexto o del instrumento para entender los resultados.

Finalmente, tras analizar lo que muestran las entrevistas y las preguntas abiertas, se concluye que las herramientas tecnologías digitales que dice utilizar el profesorado son solamente las relacionadas con el aula presencial (aula física) y el aula digital (campus virtual).

Tras las conclusiones, parece contradictorio que existan resistencias a la utilización de las tecnologías digitales pues una gran parte de los sujetos considera que las utiliza bastante. Sin embargo, aunque se observa que el profesorado conoce y utiliza las tecnologías digitales, principalmente lo hace en el aula presencial y el aula digital. Tomando como referencia a Aduviri (2012) con la clasificación de las 10 posibles aulas en las que utilizar las tecnologías digitales, se siguen utilizando las mismas que ya se utilizaban anteriormente, no parece haber una evolución. Así pues, aunque el profesorado percibe que se da una gran utilización de las tecnologías digitales, lo hace solamente con unas herramientas concretas dejando un amplio universo sin explorar.

En este sentido, el presente estudio no coincide con las conclusiones del estudio de Flores y del Arco (2012) en el que se investigó el tipo de utilización de las tecnologías digitales en la Universidad de Lleida. Tras el análisis de resultados, los autores afirmaron que "se utiliza una variedad considerable de recursos para hacer llegar la información a los estudiantes" (p.210). Sin embargo, los recursos de los que trata el estudio son mayoritariamente unidireccionales, es decir, aquellos que permiten al 
profesorado transmitir información con diferentes herramientas tecnologías digitales. Tras esta yuxtaposición con el presente estudio, se insta a reflexionar sobre la importancia de la concepción pedagógica de las tecnologías digitales. Aunque a priori los dos estudios son contradictorios, tienen resultados parecidos. Así pues, se demuestra la clara influencia del punto de vista sobre las posibilidades de las tecnologías digitales en el análisis y conclusiones en una investigación.

Uno de los problemas ha sido la propia implantación del cambio en la Facultad, que no se ha llevado de la manera más óptima para poder institucionalizarlo y generar una dinámica de actualización ante una herramienta en constante avance. Teniendo en cuenta esta situación, se concluye que la incorporación de las tecnologías digitales en la Facultad se ha tratado de un cambio y no una innovación, pues no se ha llevado a cabo el proceso necesario para ello ni tampoco se ha institucionalizado.

En cuanto al responsable de este cambio, surgen dos elementos clave: la Facultad y el profesorado o Departamentos. Se concluye que la responsabilidad debería recaer primero en las propias personas cuando éstas no utilizan al máximo los recursos que actualmente están disponibles. Por otra parte, dado que existe una parte del profesorado que exprime al máximo las infraestructuras que ofrece la Facultad, es responsabilidad de los órganos directivos la actualización de las herramientas y la promoción de su utilización.

El proceso de cambio en cuanto a la utilización generalizada de las tecnologías digitales no está institucionalizado en la Facultad de Educación. Esta puede ser una debilidad importante que explicaría la falta de utilización de las tecnologías digitales en el aula. Las fases que los diferentes autores como Curry (1992) o Gairín (2010) describen para la instauración de un cambio; o las que Sandholtz y Reilly (2004) y la organización SURF (2005, citado en Bastiaens y Zwaneveld) establecieron para el caso de la incorporación de las tecnologías digitales, no parecen haberse llevado a cabo en la Facultad de manera formal: estableciendo unos objetivos, un plan estratégico para ello, asignando un líder para el proceso de cambio, teniendo en cuenta las resistencias y preparar facilitadores, etc.

De esta manera, coincidimos con los autores anteriormente citados en que los procesos para instaurar el cambio se deben llevar a cabo para poder conseguir con éxito la implantación de la mejora. Asimismo, en el caso de la incorporación de las tecnologías digitales coincidimos con Bates (2001, en línea) en que "la utilización de la tecnología para la enseñanza no es una simple cuestión técnica (...). Las decisiones sobre la tecnología deben encuadrarse en unos objetivos educativos y supeditarse a ellos". Tal y como se ha visto en este estudio, hay una ausencia de objetivos establecidos a nivel de Facultad para conseguir la incorporación completa de las tecnologías digitales en la docencia.

\section{Referencias}

Aduviri, R. (2012). Tecnologías para el Aprendizaje y el Conocimiento. [En línea]. Recuperado de: http://www.slideshare.net/ravsirius/tecnologas-para-el-aprendizaje-y-el-conocimiento.

Bastiaens, T. y Zwaneveld, B. (2010). ICT competences of the teacher: About supporting learning and teaching processes with the use of ICT. IFIP International Federation for Information Processing, 1, 1-9.

Bates, A.W. (2001). Cómo gestionar el cambio tecnológico. Estrategias para los responsables de centros universitarios. Barcelona: Gedisa/EDIUOC.
BECTA [British Educational Communications and Technology Agency] (2004). A Review of the Research Literature on Barriers to the Uptake of ICT by Teachers. Informe. Recuperado de: http:// dera.ioe.ac.uk/1603/1/becta_2004_barrierstouptake_litrev.pdf

Bonilla-del-Río, M., Diego-Mantecón, J.M., Lena-Acebo, F.J. (2018). Estudiantes Universitarios: prosumidores de recursos digitales y mediáticos en la era de internet. Aula Abierta, 47(3), 319-326.

Butler, D. L. y Sellbom, M. (2002). Barriers to adopting technology. Educause Quarterly, 2, 22-28.

Cartagena, M. A. (2016). Uso pedagógico de Facebook y su contribución en la autoeficacia docente. Revista Latinoamericana de Tecnología Educativa, 15(1), 115-129.

Cejudo, M. (2008). Aspectos fundamentales de la formación del profesorado en TIC. Pixel-Bit: Revista de medios y educación, $31,121-130$.

Curry, B.K. (1992). Instituting enduring innovations: achieving continuity of change in higher education. ASHEERIC Higher Education Report No. 7. Washington, D.C.: The George Washington University, School of Education and Human Development. ISBN: 1-878380-20-6.

Duart, J. M. y Lupiáñez, F. (2005). Monográfico Las TIC en la universidad: estrategia y transformación. Revista de Universidad y Sociedad del Conocimiento, 2 (1), 1-109.

Falco, M. (2017). Reconsiderando las prácticas educativas: TICs en el proceso de enseñanza-aprendizaje. Tendencias Pedagógicas, 29, 59-76.

Flores, O. y Del Arco, I. (2013). Nativos digitales, inmigrantes digitales: rompiendo mitos. Un estudio sobre el dominio de las TIC en profesorado y estudiantado de la Universidad de Lleida. Bordón, 65(2), 59-74. ISSN: 0210-5934.

Gairín, J. (2010). La evaluación del impacto en programas de formación. REICE: Revista Electrónica Iberoamericana sobre Calidad, Eficacia y Cambio en Educación, 8(5), 19-43.

Gairín, J. y Rodríguez-Gómez, D. (2011). Cambio y mejora en las organizaciones educativas. Educar, 47(1), 31-50.

Gewerc, A., Montero, L. y Lama, M. (2014). Colaboración y redes sociales en la enseñanza universitaria. Revista Comunicar, 42 (21), 55-63.

Gómez, M., Roses, S. y Farias, P. (2012). El uso académico de las redes sociales en universitarios. Revista Comunicar, 38(19), 131-138.

Johnson, L., Adams Becker, S., Cummins, M., Estrada, V., Freeman, A. y Hall, C. (2016). Resumen del NMC Horizon Report: 2016 Higher Education Edition. Austin, Texas: The New Media Consortium.

Kalembera, L. y Majawa, F. (2015). The Integration of ICTs into the Learning Activities of the College of Medicine Undergraduate Students. IST-Africa 2015 Conference Proceedings (pp. 1-10). IIMC International Information Management. ISBN: 9781-905824-51-9

Lewin, K. (1947). Frontier in group dynamics. Concept, Method and Reality in Social Science; Social Equilibria and Social Change. Human relations, 1(1), 5-41.

Maor, D. y Currie, J. K (2017). The use of technology in postgraduate supervision pedagogy in two Australian universities. International Journal of Educational Technology in Higher Education, 14(1), 1-15.

Moreno, L.L. y Rochera, M.J. (2015). Congruencias y discrepancias entre concepciones y prácticas evaluativas con uso de TIC. Perspectiva Educacional. Formación de Profesores, 54(2), 126-149.

Pieterse, J.H., Caniëls, M.C.J. y Homan, T. (2012). Professional discourses and resistance to change. Journal of Organizational Change Management, 25, 798-818. 
Prendes, M. P. (2010). Competencias TIC para la docencia en la Universidad pública española: indicadores y propuestas para la definición de buenas prácticas. Informe del Proyecto EA-2009-0133 de la Secretaría de Estado y Universidades e Investigación. Recuperado de: http://www.um.es/competenciastic/informe_final_competencias2010.pdf

Prendes, M.P. y Castañeda, L. (2010). Universidades latinoamericanas ante el reto de las TIC : demandas de alfabetización tecnológica para la docencia. En Congreso Euro-Iberoamericano de Alfabetización Mediática y Culturas Digitales. Sevilla: Universidad de Sevilla. ISBN: 978-84-693-2361-8

Rodríguez-Izquierdo, R. M. (2010). El impacto de las TIC en la transformación de la enseñanza universitaria: repensar los modelos de enseñanza y aprendizaje. Teoría de la Educación: Educación y Cultura en la Sociedad de la Información, 11(1), 32-68.

Salinas, J. (2004). Innovación docente y uso de las TIC en la enseñanza universitaria. Revista universidad y sociedad del conocimiento, 1(1), 1-16.

San Martín Gutiérrez, S., Jiménez Torres N. y Jerónimo Sánchez-Beato, E. (2016). La evaluación del alumnado universitario en el Espacio Europeo de Educación Superior. Aula Abierta, 44, 7.14.
Sandholtz, J. y Reilly, B. (2004). Teachers, not technicians: Rethinking technical expectations for teachers. The Teachers College Record, 106(3), 487-512.

Sigalés, C. (2004). Formación universitaria y TIC: nuevos usos y nuevos roles. Revista Universidad y Sociedad del Conocimiento. 1(1), 1-6.

Tejedor, F. J., García-Valcárcel, A. y Prada, S. (2009). Medida de actitudes del profesorado universitario hacia la integración de las TIC. Comunicar, 33, 115-124.

UNESCO (2011). Competency Framework for Teachers. Version 2.0. Paris: United Nations Educational, Scientific and Cultural Organization. ISBN: 978-9230-01053-9.

Valdés, J. C., Ramírez, M., Moreno, H., Núñez, A. y Lugo, A. (2012). Los recursos de Internet: el papel que juegan en el proceso enseñanza-aprendizaje del nivel educativo superior. Multidisciplina, 12, 72-91.

Van Braak, J. (2001). Factors influencing the use of computer mediated communication by teachers in secondary schools. Computers \& Education, 36(1), 41-57.

Vázquez, A.I. y Cabero, J. (2015). Las redes sociales aplicadas a la formación. Revista Complutense de Educación, 26, Número especial 2015, 253-272. 